\title{
The importance of radiation for semiempirical water-use efficiency models
}

\author{
Sven Boese, Martin Jung, Nuno Carvalhais, and Markus Reichstein \\ Max Planck Institute for Biogeochemistry, Dept. for Biogeochemical Integration, \\ Hans-Knoell-Strasse 10, 07745 Jena, Germany \\ Correspondence to: Sven Boese (sboese@bgc-jena.mpg.de)
}

Received: 2 December 2016 - Discussion started: 5 January 2017

Revised: 20 April 2017 - Accepted: 30 April 2017 - Published: 22 June 2017

\begin{abstract}
Water-use efficiency (WUE) is a fundamental property for the coupling of carbon and water cycles in plants and ecosystems. Existing model formulations predicting this variable differ in the type of response of WUE to the atmospheric vapor pressure deficit of water (VPD). We tested a representative WUE model on the ecosystem scale at 110 eddy covariance sites of the FLUXNET initiative by predicting evapotranspiration (ET) based on gross primary productivity (GPP) and VPD. We found that introducing an intercept term in the formulation increases model performance considerably, indicating that an additional factor needs to be considered. We demonstrate that this intercept term varies seasonally and we subsequently associate it with radiation. Replacing the constant intercept term with a linear function of global radiation was found to further improve model predictions of ET. Our new semiempirical ecosystem WUE formulation indicates that, averaged over all sites, this radiation term accounts for up to half (39-47\%) of transpiration. These empirical findings challenge the current understanding of water-use efficiency on the ecosystem scale.
\end{abstract}

\section{Introduction}

Understanding the coupling of carbon and water cycles is a central question of global change research, as changes in one of the cycles could directly propagate to the other (Churkina et al., 1999; Gerten et al., 2004; Ito and Inatomi, 2012). Carbon assimilation through photosynthesis $(A)$ and transpiration $(T)$ constitute major fluxes in these two cycles and the Earth system (Jasechko et al., 2013; Ciais et al., 2014). On the leaf scale, water-use efficiency (WUE) is defined as
WUE $=A / T$, quantifying the ability of a plant to assimilate atmospheric carbon dioxide per water loss by transpiration. An understanding of this ratio can hence translate into the ability to predict one of the two fluxes from the other.

Both fluxes are limited by the stomatal conductance $\left(g_{\mathrm{s}}\right)$ of plant leaves (Cowan and Farquhar, 1977), allowing for the definition of intrinsic water-use efficiency, $\mathrm{WUE}_{\mathrm{i}}=A / g_{\mathrm{s}}$. This quantity is less contingent on the vapor pressure deficit of atmospheric water vapor (VPD), which affects both fluxes differently. At the ecosystem scale, neither carbon assimilation nor transpiration can be observed directly. However, the eddy covariance (EC) method (Baldocchi et al., 2001) can be used to obtain data of the gross primary productivity (GPP) and the evapotranspiration (ET). On the ecosystem scale, WUE is then defined as WUE $=\mathrm{GPP} / \mathrm{ET}$, while the intrinsic WUE is accordingly formulated as $\mathrm{WUE}_{\mathrm{i}}=\mathrm{GPP} / G_{\mathrm{s}}$, where $G_{\mathrm{S}}$ denotes the surface conductance of the ecosystem.

Analytical models that predict WUE on the leaf scale (Katul et al., 2010; Medlyn et al., 2011) were derived from theoretical optimality considerations. Corresponding variants were evaluated with ecosystem-scale flux measurements gathered by the FLUXNET ${ }^{1}$ in empirical studies (Beer et al., 2009; Zhou et al., 2014, 2015). The central difference between the existing models is the response of $g_{\mathrm{S}}$ or $G_{\mathrm{S}}$ to VPD, resulting in different dependencies of WUE on VPD. The concept of inherent water-use efficiency (IWUE) from Beer et al. (2009) corrects for the increasing diffusion of water vapor with higher values of VPD. However, the proposed IWUE is still dependent on the difference between leaf-external and leaf-internal $\mathrm{CO}_{2}$ concentrations and therefore stomatal conductance. Physiological models (e.g., Katul

\footnotetext{
${ }^{1}$ http://fluxnet.fluxdata.org/.
} 
et al., 2010) suggest stomatal contraction with increasing VPD, as plants aim to minimize water loss. This was found to be an important factor in the ecosystem scale as shown by Zhou et al. (2014) for half-hourly observations and by Zhou et al. (2015) for daily observations. In both studies, the response of WUE could be approximated to be proportional to $\mathrm{VPD}^{-0.5}$. Despite their discrepancy, both the models of Beer et al. and Zhou et al. imply that, on the ecosystem scale, WUE is strictly an inverse function of VPD or VPD ${ }^{0.5}$, respectively.

Stomatal conductance models that were derived on the plant scale include an additional conductance term $g_{0}$ that is invariable to changing levels of photosynthesis (Ball et al., 1987; Medlyn et al., 2011). Any transpiration resulting from this part of stomatal conductance should be expected to be proportional to the gradient of the partial pressure of water vapor, quantified by the atmospheric VPD observations. In contrast to formulations commonly used in stand, land surface, and vegetation models (de Kauwe et al., 2013), this process is not considered in current ecosystem-scale WUE formulations (Zhou et al., 2014, 2015).

Incoming solar radiation is a driving factor for both photosynthesis and transpiration. However, existing WUE models posit that the ratio of both is invariant with regard to this factor. This implicitly assumes that, on the ecosystem scale, the influence of radiation on GPP and ET cancels out, as the water-use efficiency is entirely determined by gas diffusion and its limiting factors. However, photosynthesis saturates at high radiation levels (Farquhar et al., 1980), even in well-watered conditions. Models of potential evapotranspiration (PET), by contrast, do not prescribe a similar limiting behavior in conditions of sufficient water availability. For example, in the Penmann-Monteith equation, the evaporation rate scales linearly with the absorbed radiation, holding all other factors constant (Leuning et al., 2008). Mechanistically, the process of equilibrium transpiration (Jarvis and McNaughton, 1986) implies that sizeable transpiration can occur even when the leaf is fully decoupled from the atmosphere, i.e., when VPD is very low. This is a second process that current ecosystem WUE models cannot accommodate.

In this study, we address these unresolved inconsistencies regarding the importance of additional model terms for predictions of ecosystem-scale transpiration. We do this empirically on the ecosystem scale by optimizing and assessing different WUE models with FLUXNET observations from 110 globally distributed towers. In our approach, ET is selected as the target variable, while the different WUE models utilize GPP as one of multiple explanatory variables. The substantial degree of correlation between GPP and ET is thus harnessed for the predictions of ET. In a first step, we identify existing biases in ecosystem-scale WUE models. In the next step, these biases are tested for their dependency on VPD and radiation. Lastly, we infer a tentative partitioning of transpiration according to its association with radiation and discuss the substantial magnitude of this metric. We conclude by highlighting how changes in the model structures impact the between-site variability in parameter estimates.

\section{Data and methods}

\subsection{Data}

The daily daytime integrals of GPP and ET were taken from the La Thuile FLUXNET (open and fair-use data policy sites) collection (La Thuile Synthesis Dataset, 2007). The aggregation to daytime values was based on values of potential radiation larger than $10 \mathrm{~W} \mathrm{~m}^{-2}$. Additionally, we used global radiation $(\mathrm{Rg})$ and the daytime VPD measured at the same EC sites.

The EC data were processed according to the standard methods (Papale et al., 2006; Reichstein et al., 2005) to assure consistent quality of the observations. Eddy covariance GPP results were based on the flux partitioning method of Reichstein (2005). We used only data with GPP $>0.1$ $g_{\mathrm{C}} \mathrm{d}^{-1} \mathrm{~m}^{-2}$, ET $>0.05 \mathrm{~mm} \mathrm{~d}^{-1}$, and VPD $>0.001 \mathrm{kPa}$ to reduce the relatively large impact of random measurement errors under low flux conditions. Following the procedure of Beer et al. (2009), we further used only data after 3 consecutive rain-free days. This reduces contributions by evaporation to the measured evapotranspiration as physical evaporation typically declines rapidly after rain events due to the depletion of water stored in the topmost soil layer (Wythers et al., 1999). That assumption similarly applies to precipitation that is intercepted on leaf surfaces and other plant parts in the canopy. As Miralles et al. (2010) summarize, the interception storage for forest ecosystems reported in different studies amounted to a mean of $1.2 \mathrm{~mm}( \pm 0.4 \mathrm{~mm}$; per unit area of canopy cover). With the mean interception evaporation rates reported as $0.3 \mathrm{~mm} \mathrm{~h}^{-1}\left( \pm 0.1 \mathrm{~mm} \mathrm{~h}^{-1}\right.$; per unit area of canopy cover), this storage can be concluded to be typically depleted within the first days after a precipitation event. Therefore, the measured evapotranspiration after 3 consecutive rain-free days is expected to approximate transpiration, and we additionally verified that our results are robust when considering longer rain-free periods (see below). For the main analysis, we included sites with at least 25 data points fulfilling the requirements noted above. A list of these 110 sites used for the parameter estimation can be found in the Supplement (Sect. S4 and Table S2 in the Supplement).

The analyses presented presume that the observed evapotranspiration is dominated by transpiration after 3 consecutive rain-free days. To test the robustness of our findings against this assumption, we varied the number of consecutive rain-free days from 0 to 14 . For each considered step, the data were filtered accordingly and parameters of the WUE models were estimated for each site. We then calculated the mean of the parameter estimates over all sites and the associated uncertainty ( $95 \%$ confidence interval, CI) of the mean via bootstrapping. We excluded some humid sites, for which 
too few data points were available, from this sensitivity analysis when filtering for longer rain-free periods. This procedure ensures that all levels of filtering for rain-free periods included the same set of sites.

\subsection{Concepts and models}

For our analysis, we started out with the WUE model of Zhou et al. (2015), which we converted for an inversion against ET data:

$\mathrm{ET}=\frac{\mathrm{GPP} \times \mathrm{VPD}^{0.5}}{\mathrm{uWUE}}$,

where uWUE represents the site-specific underlying wateruse efficiency. The introduced WUE model states that no transpiration occurs independently of GPP $\times \mathrm{VPD}^{0.5}$. To test this hypothesis, we introduced an intercept term $\left(\mathrm{ET}_{\mathrm{res}}\right)$ in the generic WUE model, which we call the $+\mathrm{ET}_{\text {res }}$ model:

$\mathrm{ET}=\frac{\mathrm{GPP} \times \mathrm{VPD}^{0.5}}{\mathrm{uWUE}}+\mathrm{ET}_{\text {res }}$.

Hence, any significant intercept would indicate transpiration that cannot be explained by GPP $\times \mathrm{VPD}^{0.5}$. The relative con-

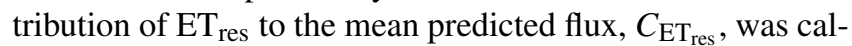
culated as

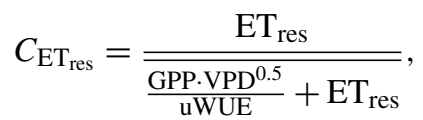

where the denominator contains the mean predicted daily ET of the model.

To further test whether $\mathrm{ET}_{\text {res }}$ relates to atmospheric variables, we postulated three different alternative models. The residual transpiration could be driven by an additional VPD term that is independent of photosynthesis, as some stomatal conductance models (Medlyn et al., 2011) already include a residual conductance term $g_{0}$, in which case the WUE model could take the form

$\mathrm{ET}=\frac{\mathrm{GPP} \times \mathrm{VPD}^{0.5}}{\mathrm{uWUE}}+g_{0} \times \mathrm{VPD}$.

We also considered the possibility that $\mathrm{ET}_{\text {res }}$ is related to global radiation $(\mathrm{Rg})$ :

$\mathrm{ET}=\frac{\mathrm{GPP} \times \mathrm{VPD}^{0.5}}{\mathrm{uWUE}}+r \times \mathrm{Rg}$.

To test for possible interactions between the two additional variables, we considered a third alternative where $\mathrm{ET}_{\text {res }}$ was modeled by including both Rg and VPD as independent factors:

$\mathrm{ET}=\frac{\mathrm{GPP} \times \mathrm{VPD}^{0.5}}{\mathrm{uWUE}}+g_{0} \times \mathrm{VPD}+r \times \mathrm{Rg}$.

In the following, we refer to the reference WUE definition (Eq. 1) as "Zhou". We abbreviate models with additional covariates by omitting the reference to the GPP VPD term $\left(\frac{\mathrm{GPP} \times \mathrm{VPD}^{0.5}}{\mathrm{uWUE}}\right)$ of Zhou, which is always used unless denoted otherwise. The model with an additional VPD term, for example, is designated as "+VPD".

\subsection{Parameter estimation and statistics}

In the following, we refer to Eqs. (1), (2), (4), (5), and (6) as models, as we optimize their fit to the EC data by estimating free parameters. The estimation was conducted with the Levenberg-Marquardt technique, minimizing the sum of squares of the model residuals. The residuals were calculated as the difference between observed and predicted ET. We used the nlsLM package in R (Elzhov et al., 2015). All parameters were restricted to positive values, preventing biologically implausible estimates. The uncertainties and correlations of the parameters were calculated with the variancecovariance matrix returned by the fitting function (Omlin and Reichert, 1999).

The model performance was assessed for each site with the Nash-Sutcliffe model efficiency (MEF; Nash and Sutcliffe, 1970):

$\mathrm{MEF}=1-\frac{\sum\left(Y_{\mathrm{prd}}-Y_{\mathrm{obs}}\right)^{2}}{\sum\left(Y_{\mathrm{obs}}-{\overline{Y_{\mathrm{obs}}}}^{2}\right.}$,

where $Y_{\text {obs }}$ are observations and $Y_{\text {prd }}$ are predictions by a model.

A MEF of 1 implies a perfect fit of the model to the data. A MEF below 0 implies that the mean of the observations outperforms the fit of the model. All MEFs were calculated in a leave-one-out cross validation to account for the problem of overfitting. Thus, the cross-validated MEFs can be used to compare models with differing numbers of free parameters.

We assessed parameter distributions stratified according to a classification of vegetation structure that was based on plant functional types (PFT). The FLUXNET sites included were classified as low-vegetation structure if the report PFT was either grassland (GRA) or crops (CRO) and high-vegetation structure if otherwise. In total, 40 sites had low-vegetation structures, compared to 70 sites with high-vegetation structures. The significance of differences between distributions of parameters and metrics for these two classes was verified by a Kolmogorov-Smirnov test (Daniel, 1990) and bootstrapped $95 \%$ confidence intervals of the mean.

\subsection{Contribution analysis}

To assess the contribution of driving variables to the predicted fluxes, we performed an analysis of attribution to the individual model terms. Consider a simple multiple linear regression model,

$Y=a_{1} \times X_{1}+a_{2} \times X_{2}$,

where $Y$ is the dependent variable, $X_{1}$ and $X_{2}$ are independent variables, and $a_{1}$ and $a_{2}$ denote the model parameters. 


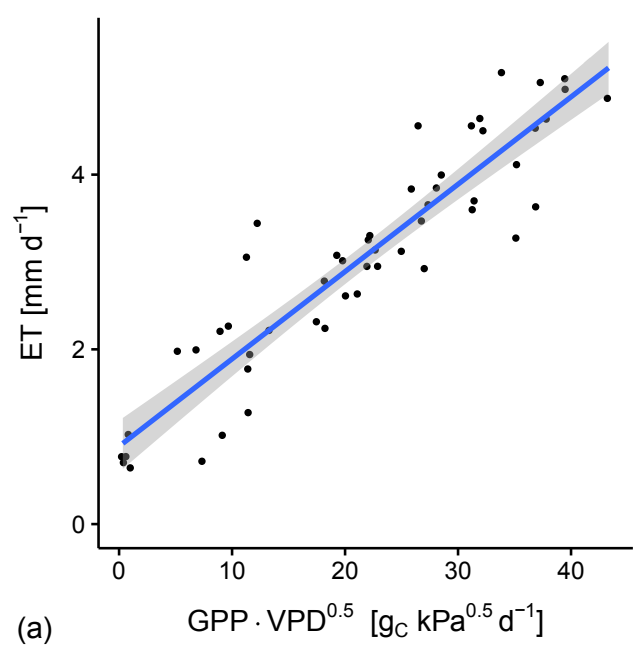

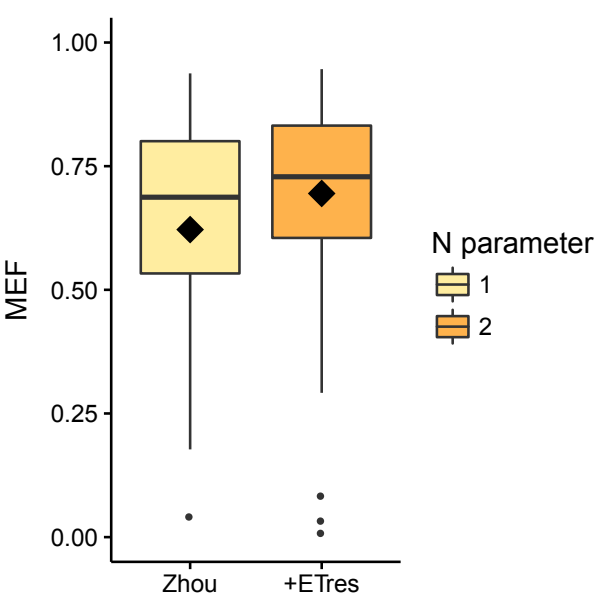

(b)

Figure 1. Linear regression between the product of GPP and VPD 0.5 and the daily ET; the shaded area indicates the $95 \%$ confidence interval of the mean of the predictions (a). Model performance of the Zhou model compared to the $+\mathrm{ET}_{\text {res }}$ variant (b). The thick horizontal lines of the box plots denote the median; the diamonds denote the mean of the MEF of all sites.

Due to the additive character of the model, the contribution of one variable (e.g., $\left.X_{1}\right)$ to the total flux is given by its product with the slope $\left(a \times X_{1}\right)$. In our analysis, we tested the contribution of the linear radiation term (Eq. 5) to the total modeled evapotranspiration. Thus, we defined the fraction of evapotranspiration that was attributed to the radiation term as

$\mathrm{ET}_{\text {frac }}=\frac{\sum(r \times \mathrm{Rg})}{\sum\left(\frac{\mathrm{GPP} \times \sqrt{\mathrm{VPD}}}{\mathrm{uWUE}}+r \times \mathrm{Rg}\right)}$.

We considered two variants to estimate this metric: parallel and hierarchical. In the first case, both parameters, uWUE and $r$, were estimated in a standard parameter estimation, i.e., concurrently. In the second case, uWUE was first estimated in the $+\mathrm{ET}_{\text {res }}$ model. We then defined a term $M$ as

$M=\mathrm{ET}_{\text {res }}+\varepsilon$,

where $\varepsilon$ denotes the residuals and $\mathrm{ET}_{\text {res }}$ denotes the intercept parameter of the $+\mathrm{ET}_{\text {res }}$ model. The parameter $r$ was then estimated in a linear regression of the form

$M=r \times \operatorname{Rg}+c$,

where $c$ denotes a constant intercept. By giving precedence to the uWUE parameter in this approach, we expect to get an estimate of a reasonable lower bound for $\mathrm{ET}_{\text {frac }}$. All parameters were constrained to positive values.

To further assess uncertainties due to problems of parameter identifiability among uWUE and $r$ in the parallel variant, we sampled 200 parameter vectors from the posterior parameter uncertainty distribution for each site.

\section{Impact of parameter correlations on the contribution analysis}

In many realistic examples, the model parameters of Eq. (8), $a_{1}$ and $a_{2}$, are not perfectly identifiable. This could be due to the correlation of $X_{1}$ and $X_{2}$ or more fundamental model structural uncertainty. In these cases, the contribution of $X_{\mathrm{i}}\left(f_{X_{\mathrm{i}}}\right)$ is confounded by the parameter correlations $a_{1}$ and $a_{2}$, leading to a high uncertainty of its estimation. In our analysis, GPP and Rg are expected to be highly correlated, leading to dependent parameter uncertainties. To evaluate this effect, we estimated the contribution of the described withinsite uncertainty of $f_{X_{\mathrm{i}}}$. The variance-covariance matrix $(V)$ of the parameter estimates could be calculated for each site with the results of the regression. Consequently, $V$ can be used to derive the respective posterior parameter distributions, from which we sampled 200 parameter vectors per site, representing the uncertainty and correlation of the two parameters. Site-specific vectors $f_{X_{\mathrm{i}}, S}$ can be calculated as

$f_{X_{\mathrm{i}}, s_{j}}=\left[f_{X_{\mathrm{i}}, s_{j}, p_{1}}, f_{X_{\mathrm{i}}, s_{j}, p_{2}}, \ldots, f_{X_{\mathrm{i}}, s_{j}, p_{200}}\right]$,

where $p_{1}$ to $p_{200}$ denote the 200 realizations of parameter vectors and $s_{j}$ denotes a specific site.

Based on this, we tested whether the global variance in $f_{X_{\mathrm{i}}}$ is a product of differing means of the site-specific $f_{X_{\mathrm{i}}, S}$ vectors or their variances. For this, we performed an ANOVA along the sites as a categorical variable with the parameter realizations as random replicates.

\section{Results}

In our analysis we tested different WUE models that predicted ET using the product of GPP and the water vapor pres- 

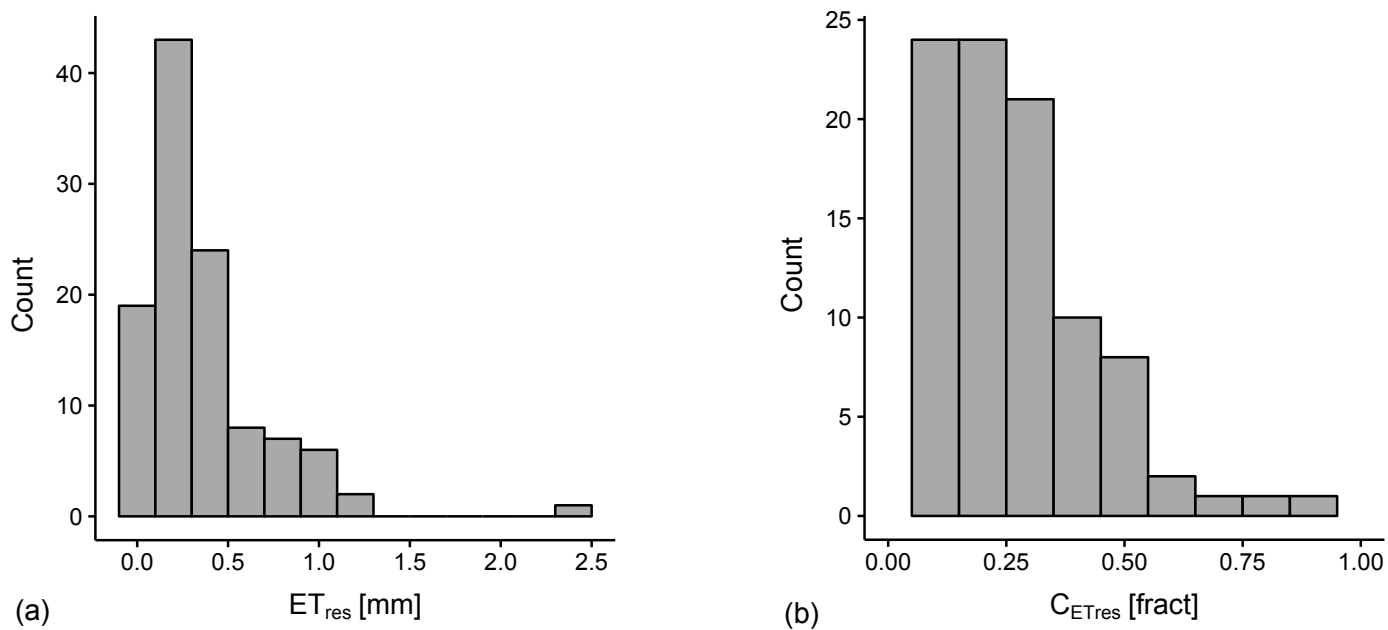

Figure 2. Distribution of the estimated intercepts. The majority of sites had values between 0 and $1.2 \mathrm{~mm}$ for the absolute intercept (a). The relative contribution of $\mathrm{ET}_{\mathrm{res}}$ to the daily mean evapotranspiration as quantified by $C_{\mathrm{ETres}}$ reached up to 0.94 (b).

Table 1. Fraction of the 110 sites at which the model of the respective row was superior to the model of the respective column according to the $\mathrm{MEF}_{\text {diff }}$ criterion.

\begin{tabular}{lrrrrr}
\hline & Zhou & + ETres & + VPD & + Rg & $+\mathrm{VPD}+\mathrm{Rg}$ \\
\hline Zhou & - & 0.14 & 0.22 & 0.07 & 0.07 \\
+ETres & 0.86 & - & 0.43 & 0.07 & 0.08 \\
+VPD & 0.78 & 0.57 & - & 0.08 & 0.08 \\
+Rg & 0.93 & 0.93 & 0.92 & - & 0.71 \\
+VPD + Rg & 0.93 & 0.92 & 0.92 & 0.29 & - \\
\hline
\end{tabular}

sure deficit, GPP $\times \operatorname{VPD}^{0.5}$, as a predictor variable. When plotting ET as a function of this multiplicative term, we observed significant intercepts, e.g., for the Mediterranean FLUXNET site IT-BCi (Fig. 1a). In these cases, significant ET was observed when the driving force of the established models, the GPP VPD product, was small or zero. When we explicitly included this term in the model (+ETres), the cross-validated MEF increased notably (Fig. 1b). As Table 1 shows, the + ETres variant outperformed the Zhou model at $86 \%$ of the sites. The respective mean difference in MEF between the two variants was 0.07 (Table 2). A corresponding table that presents the mean difference of the RMSE for all sites can be found in the supplementary materials (Table S1).

Of the 110 sites included in the analysis, $78 \%$ had a significant intercept. The site-specific estimated intercept values ranged from 0 to $2.36 \mathrm{~mm}$ ( $90 \%$ percentile $0.86 \mathrm{~mm}$ ) with a mean of $0.36 \mathrm{~mm}$ (Fig. 2a). The relative intercept $C_{\mathrm{ETres}}$ reflects the relative magnitude of the intercept to the mean daily ET of the respective sites. It ranged from 0 to $0.86(90 \%$ percentile 0.46 ) with a mean of 0.23 . This implies that circa onequarter of transpiration was not attributed to $\mathrm{GPP} \times \mathrm{VPD}^{0.5}$ in this model formulation (Fig. 2b). The importance of the intercept for the prediction of daily ET and its diverging values raises the question of whether the intercept compensates for the absence of a physical or biological process in the model or whether the observations are confounded by a systematic problem.

Our first hypothesis was that the $\mathrm{ET}_{\text {res }}$ intercept was due to the remaining contributions of soil and interception evaporation to measured ET after 3 consecutive rain-free days. To test this, we estimated the parameter $\mathrm{ET}_{\text {res }}$ for successively longer periods of consecutive rain-free days. If our hypothesis was right, we would expect a trend of declining $\mathrm{ET}_{\text {res }}$ with increasing consecutive rain-free days. However, no reduction in $\mathrm{ET}_{\text {res }}$ beyond the exclusion of the 3 days after precipitation proposed by Beer et al. (dotted line) could be observed (Fig. 3a). We therefore concluded that potential contributions of soil and interception evaporation to ET cannot explain the existence of the intercept term in the WUE model.

We then hypothesized that a missing process in the model would be discernible as a temporal pattern in the $\mathrm{ET}_{\text {res }}$ estimates. For that, we estimated the intercept for each month and site separately. The monthly means of the intercept for all sites varied in a clear seasonal pattern (Fig. 3b). ${ }^{2}$

The seasonality of $\mathrm{ET}_{\text {res }}$ suggests a relationship to meteorological variables such as VPD or radiation that vary sea-

\footnotetext{
${ }^{2}$ The monthly variability in $\mathrm{ET}_{\mathrm{res}}$ for all sites individually can be found in the Supplement (Sect. S2, Fig. S1).
} 


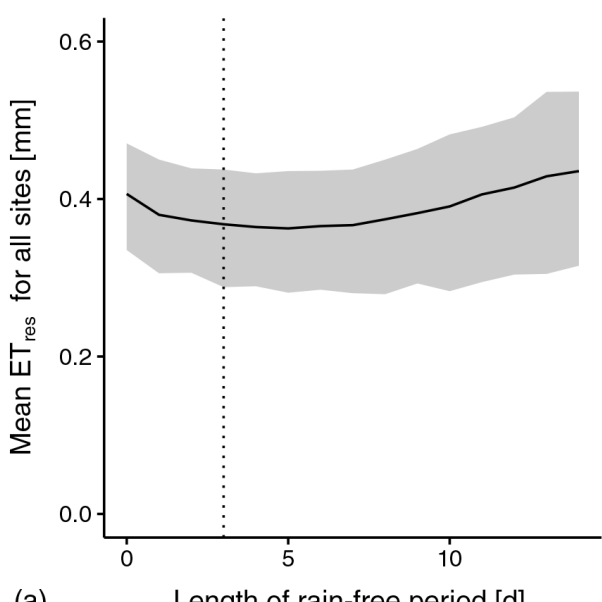

(a)

Length of rain-free period [d]

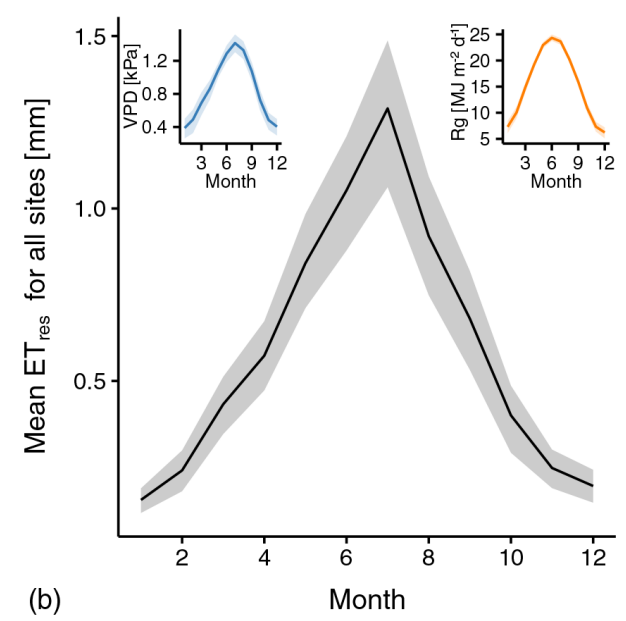

Figure 3. Sensitivity of $\mathrm{ET}_{\mathrm{res}}$ to varying length of filtering after precipitation events (a). The dotted, vertical line indicates the 3-day period we adopted from Beer et al. (2009) monthly estimation of the intercept parameter $\mathrm{ET}_{\text {res. }}$. The inset plots illustrate the mean seasonal variability in VPD and Rg for all sites on a monthly scale (b). In all plots, the solid line is the mean for all sites; the band denotes the $95 \%$ confidence interval of the mean derived by bootstrapping.

Table 2. Mean $\mathrm{MEF}_{\text {diff }}$ comparison for different model variants. Entries indicate the mean of the difference MEF(row model)-MEF(column model) for all 110 sites.

\begin{tabular}{lrrrrr}
\hline & Zhou & + ETres & + VPD & + Rg & + VPD + Rg \\
\hline Zhou & - & -0.07 & -0.09 & -0.16 & -0.17 \\
+ETres & 0.07 & - & -0.02 & -0.09 & -0.10 \\
+VPD & 0.09 & 0.02 & - & -0.07 & -0.07 \\
+Rg & 0.16 & 0.09 & 0.07 & - & 0.00 \\
+ VPD + Rg & 0.17 & 0.10 & 0.07 & 0.00 & - \\
\hline
\end{tabular}

sonally too. A relationship with VPD could represent the $\mathrm{g}_{0}$ term of canopy conductance models (Ball et al., 1987). It was therefore introduced in the form of an additional linear term (Eq. 4). A relationship with global radiation $(\mathrm{Rg})$ (Eq. 5) could represent equilibrium transpiration (Jarvis and McNaughton, 1986), where the energy surplus of incoming radiation forces a transpirational flux independent of the vapor pressure gradient. We tested modeling the $\mathrm{ET}_{\text {res }}$ intercept by including both variables separately (Eqs. 4,5$)$ and jointly (Eq. 6).

We found only a small performance increase of the +VPD variant with regard to the Zhou model with intercept term (+ETres, Fig. 4a). In fact, the $M_{\text {EF }}$ diff suggested that the $+\mathrm{ET}_{\text {res }}$ model was still superior to the $+\mathrm{VPD}$ variant at $43 \%$ of the sites (Table 1). By comparison, the $+\mathrm{Rg}$ model showed a substantial mean increase of 0.09 in the MEF compared to the $+\mathrm{ET}_{\text {res }}$ model (Table 2). The increased model complexity (two free parameters) was justified at $93 \%$ of the sites. The model variant with both VPD and Rg terms $(+\mathrm{VPD}+\mathrm{Rg})$ did not improve predictions compared to the simpler $+\mathrm{Rg}$ variant. Plots showing the impact of the $+\mathrm{ETr}_{\mathrm{es}}$ and $+\mathrm{Rg}$ variants on the accuracy of the ET predictions for two selected sites can be found in the Supplement (Sect. S3, Figs. S2, S3).

We also tested whether the variation in ET that was unexplained by the $+\mathrm{Rg}$ model was still correlated with radiation. This would indicate that the chosen linear radiation term did not fully account for all covariation between ET and $\mathrm{Rg}$, implying a nonlinear dependency. The mean $R^{2}$ between the residuals of the $+\mathrm{Rg}$ variant and observed ET was $3.9 \%$ (maximum: $22 \%, 90 \%$ percentile: $10 \%$ ). This suggests that the linear variant was an adequate choice, as little of the unexplained variation in ET was still correlated with radiation.

The difference between the $+\mathrm{Rg}$ and + VPD models is equally distinct, when the presence of a remaining model bias was tested. For this, the intercept was estimated with all four model variants (Zhou, $+\mathrm{VPD},+\mathrm{Rg}$, and $+\mathrm{VPD}+\mathrm{Rg}$ ). Only the models with an $\mathrm{Rg}$ term had considerably reduced residual intercepts (Fig. 4b).

All models compared to the original definition Zhou had two or more parameters. Of those models with two parameters, the $+\mathrm{Rg}$ emerged as the best model after cross validation, indicating that the additional model complexity was in fact justified. The presence of two parameters raised the question of whether they could be identified independently. 


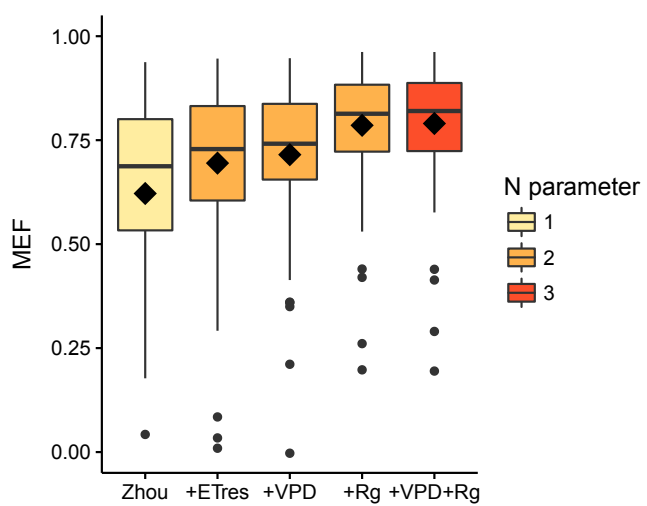

(a)

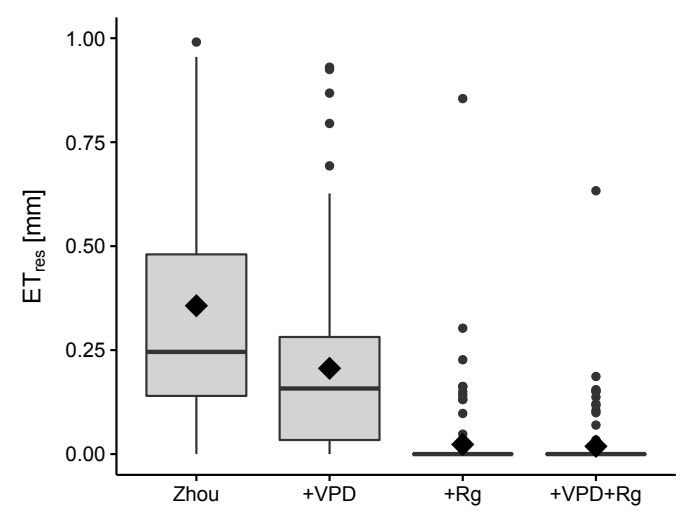

(b)

Figure 4. Cross-validated MEFs of the model variants with additional covariates (a). The thick horizontal lines of the box plots denote the median and the diamonds denote the mean of the MEF of all sites. The $+\mathrm{Rg}$ variant led to a further increase in model performance when comparing to the $+\mathrm{ET}_{\text {res }}$ variant. By contrast, the $+\mathrm{VPD}$ variant showed only a small increase in performance. Global distribution of the residual intercept for all model variants $(\mathbf{b})$.

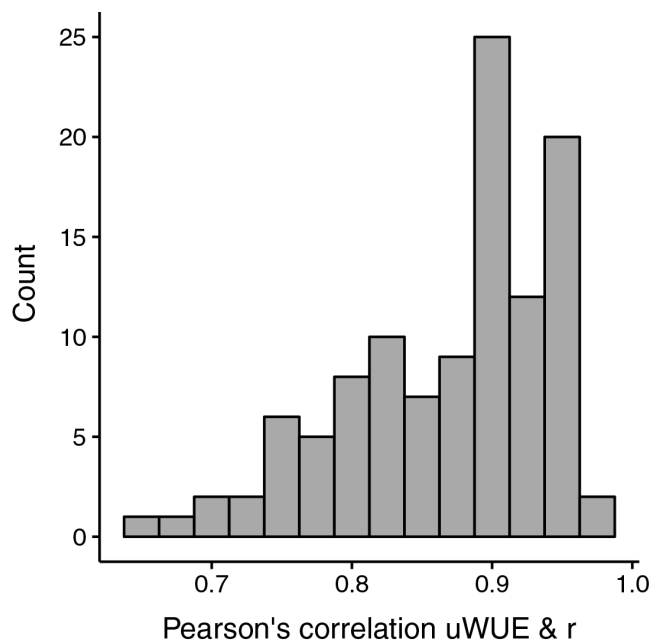

Figure 5. Correlation of the parameter estimates for $r$ and uWUE across sites.

In fact, we found a high degree of correlation between the parameters for all sites (Fig. 5). It is likely that the correlation originates from the correlation between GPP and Rg.

Parameter distributions were separated for low- and highvegetation structures. The estimates of uWUE (Fig. 6a) were not significantly different for low- and high-vegetation structures (Kolmogorov-Smirnov test, $p=0.35$ ). By contrast, the estimates for $r$ (Fig. 6b) were significantly higher for low(mean: $0.054,95 \%$ CI: 0.047-0.062) compared to highvegetation structures (mean: 0.041, $95 \%$ CI: 0.034-0.049; Kolmogorov-Smirnov test, $p=0.003$ ).

As transpiration of the $+\mathrm{Rg}$ model is a linear combination of stomatal and radiation-driven components, it is possible to calculate the relative contribution of each component to daily ET fluxes. We compared two approaches to calculating this quantity: parallel for estimating both parameters concurrently and hierarchical for estimating $r$ only after uWUE has been calibrated (Eqs. 10, 11). In both approaches, we observed that a sizeable fraction of mean daily ET could be attributed to the radiation term (Fig. 7a). For the hierarchical approach, the mean global $\mathrm{ET}_{\text {frac }}$ was $24 \%$ (95\% CI: $21-$ $27 \%$ ); for the parallel approach, the mean global $\mathrm{ET}_{\text {frac }}$ was $43 \%$ (95\% CI: 39-47\%). Similar to the assessment of parameter distributions, we stratified the $\mathrm{ET}_{\text {frac }}$ index for lowand high-vegetation structures (Fig. 7b). $\mathrm{ET}_{\text {frac }}$ was significantly higher for low- (mean: 0.53 ; $95 \%$ CI: 0.48-0.58) compared to high-vegetation structures (mean: 0.38 ; $95 \%$ CI: 0.33-0.43; Kolmogorov-Smirnov test $p<0.001$ ).

Notably, $\mathrm{ET}_{\text {frac }}$ estimated with the parallel approach varied widely between the sites (Fig. 8a). We assessed to which degree this variability can be interpreted as between-site variability in the expected value of $\mathrm{ET}_{\text {frac }}$ or whether it is due to poorly constrained and correlated parameters due to co-linearity (Fig. 5). The within-site uncertainty of $\mathrm{ET}_{\text {frac }}$ caused by parameter uncertainty was quantified as the range between the 97.5 and the 2.5 percentiles ( $95 \%$ confidence interval) of $\mathrm{ET}_{\text {frac }}$ estimates from 200 parameter vectors sampled from their respective posterior distributions. The vast majority of sites had a CI lower than 0.3 (Fig. 8b). This suggests that the large variability in $\mathrm{ET}_{\text {frac }}$ was not a result of parameter uncertainties. The conducted ANOVA supported this conclusion, as it revealed that $96 \%$ of the global $\mathrm{ET}_{\text {frac }}$ variability could be attributed to the variability between sites.

Finally, we assessed the impact of $\mathrm{ET}_{\text {res }}$ and $\mathrm{Rg}$ as additional covariates on the global variability in uWUE estimates (Fig. 9). We calculated Kendall's $\tau$ rank correlation coefficient (Kendall, 1938) between the site-level estimates of uWUEs derived from the Zhou model and two different variants: $+\mathrm{ET}_{\text {res }}$ and $+\mathrm{Rg}$. The degree of correlation of the uWUE estimates quantifies whether changes in the model 

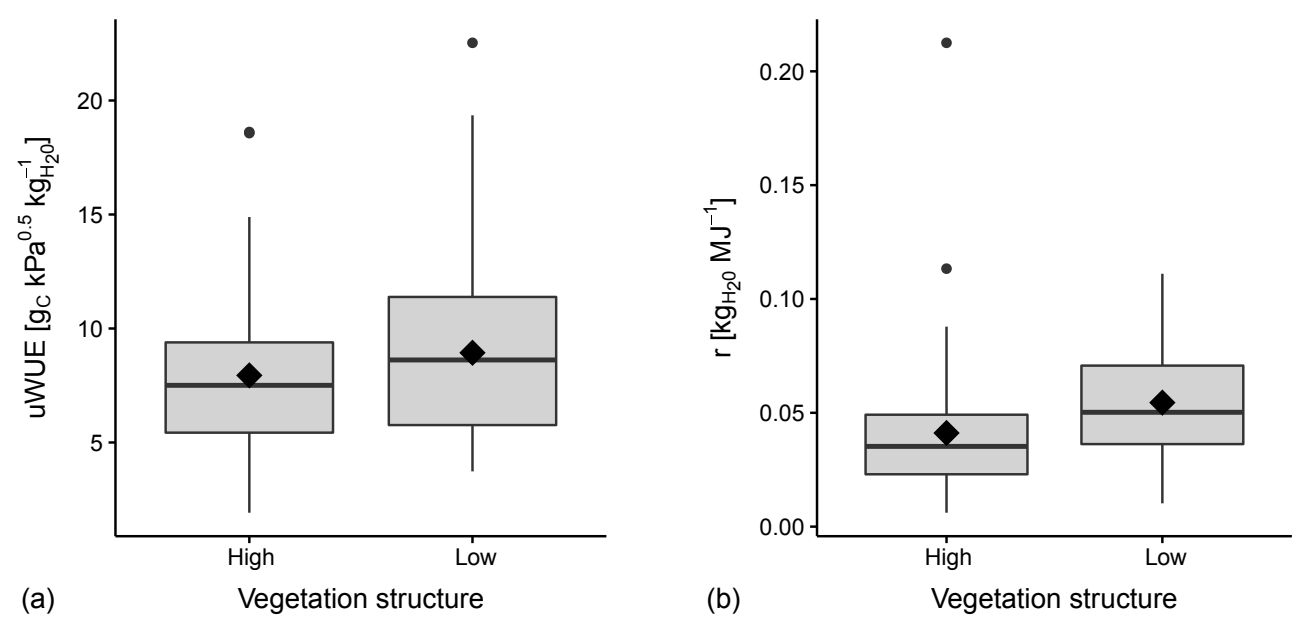

Figure 6. Distribution of the parameter estimates partitioned by type of vegetation structure. For uWUE (a), a Kolmogorov-Smirnov test yielded no difference in the distribution between the two classes of vegetation structure $(p=0.35)$. For $r$ (b), the same test indicated a difference in the distribution between the two classes of vegetation structure $(p=0.003)$.
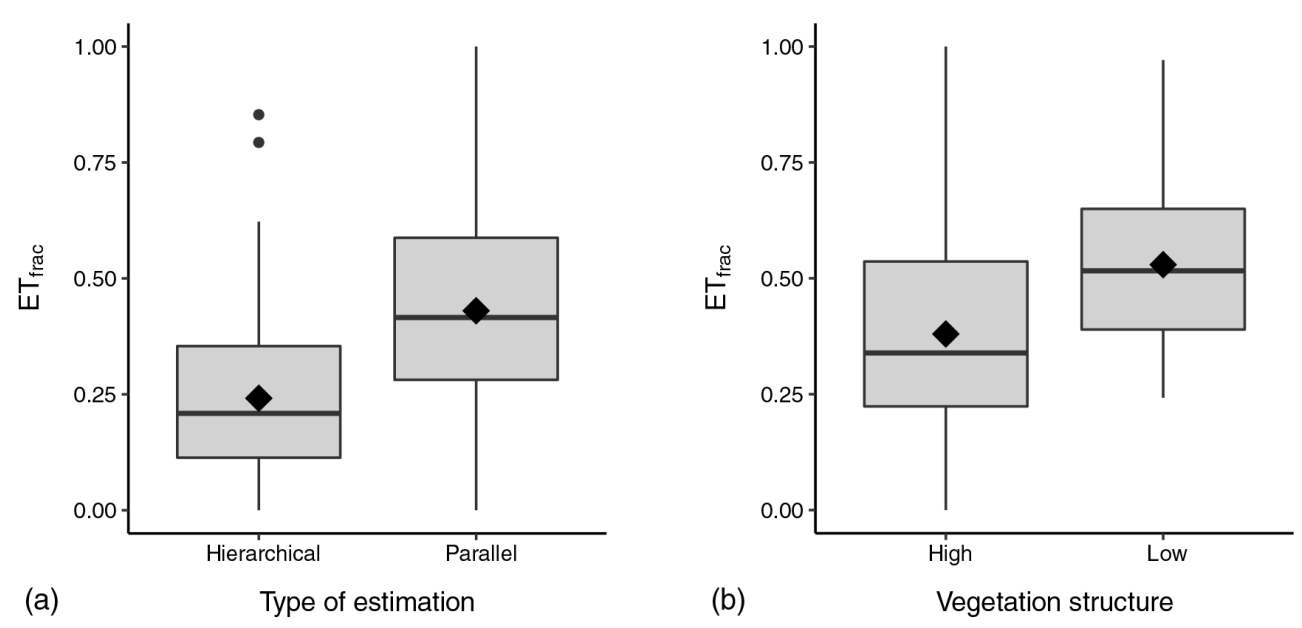

Figure 7. Distributions of $\mathrm{ET}_{\mathrm{frac}}$ estimates for all sites. The diamonds indicate the mean and the bold horizontal lines indicate the median. The hierarchical approach yielded substantially lower values and can be interpreted as a conservative estimate of the quantity (a). $\mathrm{ET}_{\text {frac }}$ was significantly higher at sites with low-vegetation structure (b).

structure permutes the ordering of the estimates between sites. The extent to which parameter estimates are affected by the model structure is crucial because any explanation or prediction of parameter values between sites would be highly desirable. While a moderate correlation between the uWUE estimates of the Zhou model with the $+\mathrm{ET}_{\text {res }}$ variant can be seen $(\tau=0.76)$, the correlation of the uWUE estimates of the Zhou model with the respective values of the $+\mathrm{Rg}$ variant is low $(\tau=0.48)$. The between-site variability in uWUE explained by the estimates of the Zhou variant was $79 \%$ for the $+\mathrm{ET}_{\text {res }}$ variant and $31 \%$ for the $+\mathrm{Rg}$ variant, as quantified by the $R^{2}$.

\section{Discussion}

\subsection{Findings and mechanisms}

In this study, we identified radiation as an important variable for ecosystem-scale transpiration and water-use efficiency. Depending on the approach used, we attributed between onequarter and one-half of mean daily transpiration of all included FLUXNET sites to a linear radiation term. These findings raise the question of which biophysical or ecophysiological processes can account for the estimated magnitudes of this attributed fraction.

The influence of radiation on stomatal conductance has been noted and discussed in the literature (Whitehead et al., 1981; Jarvis and McNaughton, 1986) and is also reflected 

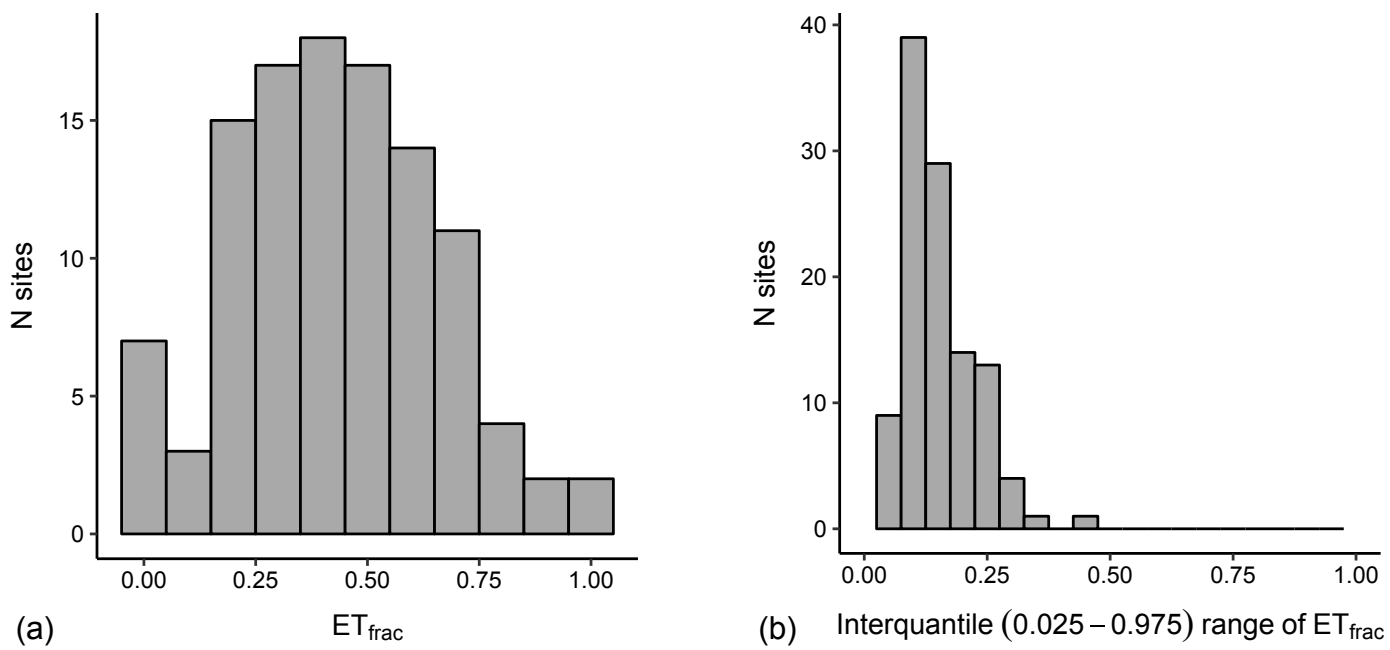

Figure 8. Distribution of the fraction of mean daily transpiration $\left(\mathrm{ET}_{\mathrm{frac}}\right)$ attributed to the radiation term for all sites, calculated with the parallel approach (a). Distribution of the within-site variability in $\mathrm{ET}_{\text {frac }}$, calculated as the difference between the 97.5 and 2.5 percentiles of the $\mathrm{ET}_{\text {frac }}$ estimates derived by sampling from the posterior parameter densities parallel approach (b).

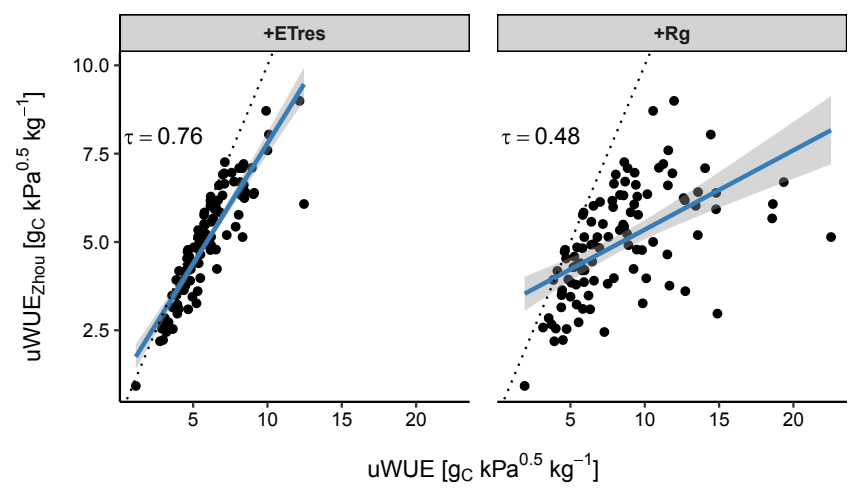

Figure 9. Effect of different model variants on global uWUE estimates. The $1: 1$ line is dotted. A linear dependency would indicate that uWUE estimates are commensurable across sites, even if only after a linear scaling. When comparing with the estimates of the original Zhou model, the correlation is notably weaker for $+\mathrm{Rg}$ than for $+\mathrm{ET}_{\text {res }}$. Sites with high parameter uncertainties were removed for all models.

in existing transpiration models (Leuning et al., 2008). By contrast, we detected a substantial transpiration component that was statistically independent of the product of GPP and $\mathrm{VPD}^{0.5}$, tentatively suggesting an insensitivity to stomatal conductance. Our results suggest that this additional flux could not be associated with a $g_{0}$ conductance term, as this would imply the dependency of the additional ET on a linear VPD term (Ball et al., 1987). By contrast, the intercept was shown to be more consistent with radiation. Consequently, models that integrated radiation-driven transpiration with such an additive linear response had a superior predictive performance across flux towers.
The observed effect of radiation indicates that equilibrium transpiration could play an important role in ecosystemscale transpiration. Equilibrium transpiration (or equilibrium evaporation rate) is the transpiration occurring if the leaf is completely decoupled from the atmosphere (Jarvis and McNaughton, 1986). Therefore, equilibrium transpiration is independent of stomatal conductance and driven solely by the dissipation of energy, provided by incoming solar radiation. According to McNaughton and Jarvis (1983), the decoupling parameter $\Omega$ is notably higher in grasslands compared to forests. This parameter reflects the proportion of evaporation that is independent of the driving gradient in the water vapor pressure. Our observation that both the radiation parameter $r$ and the index $\mathrm{ET}_{\text {frac }}$ were significantly higher for ecosystems with low-vegetation structure is therefore consistent with the explanation that equilibrium evaporation is responsible for the observed role of radiation.

This can be contrasted with a competing explanation that the radiation dependency of transpiration does not reflect an additional process but rather a systematic problem of the VPD observations. VPD is measured together with the fluxes above the canopy. While the recorded water and carbon fluxes do in fact represent the net fluxes of the tower footprint, the same cannot be said for VPD. Its measurement above the canopy may differ substantially from the relevant magnitude of the variable on the leaf scale. However, leaf temperature and thus the VPD of the leaf boundary layer are dependent on solar radiation (Tenhunen et al., 1990). Adding solar radiation to the equation could therefore be seen as compensating for the lack of the aforementioned leaf-scale VPD observations. 


\subsection{Limitations}

One limitation stems from the selection of rain-free periods for the parameter estimation. As previously described, this is a necessary step to justifiably assume that the observed latent heat fluxes constitute mostly a transpiration flux rather than evaporation from bare soil and leaf surfaces in addition to transpiration. It also makes our work comparable with the study of Beer et al. (2009), who used this method to derive their estimates. For the observed residual evapotranspiration $\mathrm{ET}_{\text {res }}$ we could show that it is not an artefact of insufficient exclusion of days after precipitation events. However, the environmental conditions during and after rain events generally represent some of these specific conditions: low VPD due to the moisture available for evaporation, a higher share of diffuse radiation, and a more or less sudden increase in soil moisture, among others. All variables mentioned could plausibly be assumed to have an influence on the stomatal opening of plants. Therefore, the WUE model presented must not necessarily predict flux relationships during or immediately after precipitation events due to the underrepresentation of similar conditions in the sample of observations used for parameter estimation.

The effect of atmospheric $\mathrm{CO}_{2}$ concentrations on WUE (Morison, 1985; Conley et al., 2001) was not considered in this analysis. One could suspect that the seasonal variability in $\mathrm{ET}_{\text {res }}$ is affected by seasonal $\mathrm{CO}_{2}$ variability if both were in phase. However, $\mathrm{ET}_{\text {res }}$ showed a global maximum during June-July, while northern hemispheric $\mathrm{CO}_{2}$ concentrations are at their minimum in September-October (Keeling et al., 1976), implying that $\mathrm{CO}_{2}$ concentrations are unlikely to cause the seasonal variation in $\mathrm{ET}_{\text {res }}$.

The presented results are subject to the eco-climatological representativeness of the FLUXNET in general and sites with a fair and free-use data policy specifically. This means that tropical areas in particular are underrepresented in our analysis (with only one Australian site, AU-How). However, all posited mechanisms that could be responsible for the observed effect of radiation are not expected to be restricted to extratropical regions. Further research is however required to quantify the importance of radiation for water-use efficiency and transpiration in tropical ecosystems.

The model structures we tested in this study were evaluated according to their global empirical adequacy. Thus, despite the possible identification of probable mechanisms responsible for the observed patterns, the model structure selected in the end likely does not reflect the exact physical mechanisms by which the ecosystem operates. Furthermore, this limitation can be specifically important in the case of water limitation. As the $\mathrm{Rg}$ term is completely independent of any variable reflecting vegetation activity, our model would predict transpiration scaling with radiation during periods of severe drought. By principle, the same problem could affect periods of low temperatures before leaf flushing, although these periods are generally also associated with low radiation levels and hence may not be as problematic.

\subsection{Implications}

Our empirical analysis suggests that ecosystem-scale transpiration depends to a sizeable degree on radiation rather than only the products GPP and VPD ${ }^{0.5}$. This implies that photosynthesis and transpiration might be less strongly coupled on the ecosystem scale than commonly assumed. We speculate that the additional effect of radiation could be due to equilibrium transpiration where radiation drives transpiration even when the canopy is fully decoupled from the atmosphere. The evidence provided for the differences between low- and high-vegetation structures gives additional credence to this explanation. However, we cannot disentangle direct physiological effects of radiation on transpiration from other radiation effects that are relevant on the ecosystem scale at this point. For the latter, leaf to canopy scaling, micrometeorological conditions, and boundary layer dynamics might contribute to the observed relationship between ecosystemscale water-use efficiency and radiation. Thus, further research is needed to reconcile our empirical findings with detailed ecosystem-scale modeling and theory, on the one hand, and plant physiology research under controlled conditions on the other hand.

Finally, we caution against prematurely interpreting the between-site variability in underlying water-use efficiency (uWUE). We showed that estimates of uWUE derived with the Zhou model explained only one-third of the observed variability in ecosystem uWUE derived from our empirically superior model formulation. The dependence of uWUE estimates on the chosen model formulation makes the interpretation of uWUE as an ecosystem property problematic. This concern would also hold for assessing temporal dynamics of uWUE such as long-term trends. For example, the unexpectedly large global trend of WUE across FLUXNET sites (Keenan et al., 2013) would need to be tested for its omission of radiation in the model that was used. Overall, this study highlights the importance of model structure uncertainty for interpretations of parameter variability.

Data availability. For this study, we used observations of the FLUXNET initiative from sites with an open and fair-use data policy (FLUXNET Data Download, 2007).

\section{The Supplement related to this article is available online at https://doi.org/10.5194/bg-14-3015-2017-supplement.}

Competing interests. The authors declare that they have no conflict of interest. 
Acknowledgements. This work used eddy covariance data acquired by the FLUXNET community and in particular by the following networks: AmeriFlux (US Department of Energy, Biological and Environmental Research, Terrestrial Carbon Program (DEFG02-04ER63917 and DE-FG02-04ER63911)), AfriFlux, AsiaFlux, CarboAfrica, CarboEuropeIP, CarboItaly, CarboMont, ChinaFlux, Fluxnet-Canada (supported by CFCAS, NSERC, BIOCAP, Environment Canada, and NRCan), GreenGrass, KoFlux, LBA, NECC, OzFlux, TCOS-Siberia, and USCCC. We acknowledge the financial support for the eddy covariance data harmonization provided by CarboEuropeIP, FAO-GTOS-TCO, iLEAPS, Max Planck Institute for Biogeochemistry, the National Science Foundation, the University of Tuscia, Université Laval and Environment Canada and the US Department of Energy. Furthermore, we thank the database development and technical support from the Berkeley Water Center, Lawrence Berkeley National Laboratory, Microsoft Research eScience, Oak Ridge National Laboratory, the University of California - Berkeley, and the University of Virginia.

We are grateful to Anke Hildebrandt for discussion and feedback on the paper.

The article processing charges for this open-access publication were covered by the Max Planck Society.

Edited by: A. Ito

Reviewed by: three anonymous referees

\section{References}

Baldocchi, D., Falge, E., Gu, L., Olson, R., Hollinger, D., Running, S., Anthoni, P., Bernhofer, C., Davis, K., Evans, R., Fuentes, J., Goldstein, A., Zhou, G., Law, B., Lee, X., Malhi, Y., Meyers, T., Munger, W., Oechel, W., Paw U,K.T., Pilegaard, K., Schmid, H., Valentini, R., Verma, S., Vesala, T., Wilson, K., and Wofsy, S.: FLUXNET: A new tool to study the temporal and spatial variability of ecosystem-scale carbon dioxide, water vapor, and energy flux densities, B. Am. Meteorol. Soc., 82, 2415-2434, 2001.

Ball, J. T., Woodrow, I. E. and Berry, J. A.: A Model Predicting Stomatal Conductance and its Contribution to the Control of Photosynthesis under Different Environmental Conditions, in: Progress in Photosynthesis Research, edited by: Biggins, J., Springer Netherlands, Dordrecht, 221-224, 1987.

Beer, C., Ciais, P., Reichstein, M., Baldocchi, D., Law, B. E., Papale, D., Soussana, J.-F., Ammann, C., Buchmann, N., Frank, D., Gianella, D., Janssens, I. A., Knohl, A., Köstner, B., Moors, E., Roupsard, O., Verbeeck, H., Vesala, T., Williams, C. A., and Wohlfahrt, G.: Temporal and among-site variability of inherent water use efficiency at the ecosystem level, Global Biogeochem. Cy., 23, GB2018, https://doi.org/10.1029/2008GB003233, 2009.

Churkina, G., Running, S. W., and Schloss, A. L.: Comparing global models of terrestrial net primary productivity (NPP): the importance of water availability, Glob. Change Biol., 5, 46-55, 1999.

Ciais, P., Sabine, C., Bala, G., Bopp, L., Brovkin, V., Canadell, J., Chhabra, A., DeFries, R., Galloway, J., Heimann, Jones, C., Le Quéré, C., Myneni, R. B., Piao, S., and Thornton, P.: Carbon and Other Biogeochemical Cycles, in: Climate Change 2013: The Physical Science Basis, Contribution of Working Group I to the Fifth Assessment Report of the Intergovernmental Panel on Climate Change, edited by: Stocker T. F., Qin D., Plattner G. K., Tignor M., Allen S. K., Boschung J., Nauels A., Xia Y., Bex V., and Midgley P. M., Cambridge University Press, Cambridge, United Kingdom and New York, NY, USA, 465-570, 2014.

Conley, M. M., Kimball, B., Brooks, T., Pinter, P., Hunsaker, D., Wall, G., Adam, N., LaMorte, R., Matthias, A., Thompson, T. L., Leavitt, S. W., Ottman, M. J., Cousins, A. B., and Triggs, J. $\mathrm{M}$.: $\mathrm{CO}_{2}$ enrichment increases water-use efficiency in sorghum, New Phytol., 151, 407-412, 2001.

Cowan, I. R. and Farquhar, G. D.: Stomatal function in relation to leaf metabolism and environment, in: Integration of Activity in the Higher Plant, edited by Jennings, D. H., Cambridge University Press, 471-505, 1977.

Daniel, W. W.: Applied Nonparametric Statistics. PWS-Kent, Boston, 1990.

de Kauwe, M. G., Medlyn, B. E., Zaehle, S., Walker, A. P., Dietze, M. C., Hickler, T., Jain, A. K., Luo, Y., Parton, W.J ., Prentice, I. C., and Smith, B. Forest water use and water use efficiency at elevated $\mathrm{CO}_{2}$ : a model-data intercomparison at two contrasting temperate forest FACE sites, Glob. Change Biol., 19, 1759-1779, 2013.

Elzhov, T. V., Mullen, K. M., Spiess, A. N., and Bolker, B.: minpack.lm: R Interface to the Levenberg-Marquardt Nonlinear Least-Squares Algorithm Found in MINPACK, Plus Support for Bounds, R package version 1.2-0, 2015.

Farquhar, G. D., von Caemmerer, S., and Berry, J. A.: A biochemical model of photosynthetic $\mathrm{CO}_{2}$ assimilation in leaves of $\mathrm{C}_{3}$ species, Planta, 149, 78-90, 1980.

FLUXNET Data Download: http://www.fluxdata.org/ DataDownload/default.aspx (last access: 8 June 2017), 2007.

Gerten, D., Schaphoff, S., Haberlandt, U., Lucht, W., and Sitch, S.: Terrestrial vegetation and water balance - hydrological evaluation of a dynamic global vegetation model, J. Hydrol., 286, 249270, 2004.

Ito, A. and Inatomi, M.: Water-use efficiency of the terrestrial biosphere: a model analysis focusing on interactions between the global carbon and water cycles, J. Hydrometeorol., 13, 681-694, 2012.

Jarvis, P. G. and McNaughton, K.: Stomatal control of transpiration: scaling up from leaf to region, Adv. Ecol. Res., 15, 1-49, 1986.

Jasechko, S., Sharp, Z. D., Gibson, J. J., Birks, S. J., Yi, Y., and Fawcett, P. J.: Terrestrial water fluxes dominated by transpiration, Nature, 496, 347-351, 2013.

Katul, G., Manzoni, S., Palmroth, S., and Oren, R.: A stomatal optimization theory to describe the effects of atmospheric $\mathrm{CO}_{2}$ on leaf photosynthesis and transpiration, Ann. Bot.-London, 105, 431-442, 2009.

Keeling, C. D., Bacastow, R. B., Bainbridge, A. E., Ekdahl, C. A., Guenther, P. R., Waterman, L. S., and Chin, J. F.: Atmospheric carbon dioxide variations at Mauna Loa observatory, Hawaii, Tellus, 28, 538-551, 1976.

Keenan, T. F., Hollinger, D. Y., Bohrer, G., Dragoni, D., Munger, J. W., Schmid, H. P., and Richardson, A. D.: Increase in forest water-use efficiency as atmospheric carbon dioxide concentrations rise, Nature, 499, 324-327, 2013.

Kendall, M.: A New Measure of Rank Correlation, Biometrika, 30, 81-89, 1938.

La Thuile Synthesis Dataset: http://fluxnet.fluxdata.org/data/ la-thuile-dataset/ (last access: 8 June 2017), 2007. 
Leuning, R., Zhang, Y., Rajaud, A., Cleugh, H., and Tu, K.: A simple surface conductance model to estimate regional evaporation using MODIS leaf area index and the Penman-Monteith equation, Water Resour. Res., 44, https://doi.org/10.1029/2007WR006562, 2008.

McNaughton, K. G. and Jarvis, P. G.: Predicting effects of vegetation changes on transpiration and evaporation, in: Water Deficits and Plant Growth, edited by: Kozlowski, T. T., Academic Press, New York, 7, 1-47, 1983.

Medlyn, B. E., Duursma, R. A., Eamus, D., Ellsworth, D. S., Prentice, I. C., Barton, C. V. M., Crous, K. Y., de Angelis, P., Freeman, M., and Wingate, L.: Reconciling the optimal and empirical approaches to modelling stomatal conductance, Glob. Change Biol., 17, 2134-2144, 2011.

Miralles, D. G., Gash, J. H., Holmes, T. R. H., de Jeu, R. A. M., and Dolman, A. J.: Global canopy interception from satellite observations, J. Geophys. Res., 115, D16122, https://doi.org/10.1029/2007WR006562, 2010.

Morison, J. I.: Sensitivity of stomata and water use efficiency to high $\mathrm{CO}_{2}$, Plant Cell Environ., 8, 467-474, 1985.

Nash, J. E. and Sutcliffe, J. V.: River flow forecasting through conceptual models part I - A discussion of principles, J. Hydrol., 10, 282-290, 1970.

Omlin, M. and Reichert, P.: A comparison of techniques for the estimation of model prediction uncertainty, Ecol. Model., 115, 45-59, 1999.

Papale, D., Reichstein, M., Aubinet, M., Canfora, E., Bernhofer, C., Kutsch, W., Longdoz, B., Rambal, S., Valentini, R., Vesala, T., and Yakir, D.: Towards a standardized processing of Net Ecosystem Exchange measured with eddy covariance technique: algorithms and uncertainty estimation, Biogeosciences, 3, 571-583, https://doi.org/10.5194/bg-3-571-2006, 2006.
Reichstein, M., Falge, E., Baldocchi, D., Papale, D., Aubinet, M., Berbigier, P., Bernhofer, C., Buchmann, N., Gilmanov, T., Granier, A., Grünwald, T., Havránková, K., Ilvesniemi, H., Janous, D., Knohl, A., Laurila, T., Lohila, A., Loustau, D., Matteucci, G., Meyers, T., Miglietta, F., Ourcival, J.-M., Pumpanen, J., Rambal, S., Rotenberg, E., Sanz, M., Tenhunen, J., Seufert, G., Vaccari, F., Vesala, T., Yakir, D., and Valentini, R.: On the separation of net ecosystem exchange into assimilation and ecosystem respiration: review and improved algorithm, Glob. Change Biol., 11, 1424-1439, 2005.

Tenhunen, J., Serra, A. S., Harley, P., Dougherty, R., and Reynolds, J. F.: Factors influencing carbon fixation and water use by Mediterranean sclerophyll shrubs during summer drought, Oecologia, 82, 381-393, 1990.

Wythers, K. R., Lauenroth, W. K., and Paruelo, J. M.: Bare-soil evaporation under semiarid field conditions, Soil Sci. Soc. Am. J., 63, 1341-1349, 1999.

Zhou, S., B. Yu, Y. Huang, and Wang, G.: The effect of vapor pressure deficit on water use efficiency at the subdaily time scale, Geophys. Res. Lett., 41, 5005-5013, 2014.

Zhou, S., Yu, B., Huang, Y., and Wang, G.: Daily underlying water use efficiency for AmeriFlux sites, J. Geophys. Res.-Biogeo., 120, 887-902, 2015. 